\title{
Phase inversion in two-phase solid systems driven by an elastic modulus mismatch
}

\author{
M. P. Gururajan*† and T. A. Abinandanan \\ Department of Materials Engineering, Indian Institute of Science, Bangalore 560012 INDIA \\ ${ }^{\dagger}$ Department of Materials Science and Engineering, Northwestern University, Evanston, IL 60208 USA
}

(20 March 2007)

\begin{abstract}
We have used phase field simulations to study elastic stress driven phase inversion in which an initial microstructure with a minority phase embedded in a majority phase evolves to one in which the latter becomes embedded in the former. Such phase inversion is possible if the majority phase is elastically stiffer than the minority phase. For a given set of parameters (volume fraction and elastic moduli of the phases), phase inversion occurs at a characteristic microstructural length scale $\left(\ell^{c}\right)$. Our results show that $\ell^{c}$ is lower for systems with larger mismatch in elastic moduli, and (to a smaller extent) in those with greater elastic anisotropy.
\end{abstract}

\section{Introduction}

A typical two phase microstructure consists of a topologically continuous 'matrix' phase in which islands of 'precipitate' phase are embedded. Usually, the matrix phase is also the majority phase in terms of volume fraction. For example, simulations of spinodal decomposition in off-symmetric alloys always lead to microstructures in which the minority phase is embedded within the majority phase.

In solid systems with a dilatational or isotropic lattice paramter mismatch, however, elastic energy considerations [1,2] lead us to expect the hard phase to assume compact (or, precipitate-like) forms embedded in the softer phase. In elastically isotropic systems, for example, Barnett et al [1] showed that elastic energy minimizing shape of an isolated particle is a sphere for a hard particle and a thin plate for a soft particle. Thus, if the early-stage microstructure has a soft (minority) phase embedded in a hard matrix, it is expected to assume elongated shapes with increasing size; if its volume fraction is sufficiently large, the elongated plates of soft phase could coalesce to form a percolating network. In other words, even though the soft phase has a lower volume fraction, it becomes the 'matrix' in which the hard, majority phase becomes embedded. We term this phenomenon 'phase inversion'.

Phase inversion is known to occur in polymer blends due to differences in viscosity of the two phases which, in turn, leads to different flow behaviours [3, 4]. Analogously, in solid systems, it is driven by differences in elastic moduli. While it has not been observed experimentally, it has been shown to be possible in several simulation studies [5-7]. These studies, however, are limited by (a) their use of isotropic elastic moduli, (b) approximations used for computing elastic stresses, and (c) small system sizes. Further, in the studies by Onuki and Nishimori [5] and Sagui et al [6], spinodal decomposition in different systems (for example, with different values for elastic modulus mismatch) leads to different 'initial' microstructures (which then undergo phase inversion at later stages). Since the initial microstructures are different, their simulations do not allow a systematic study of phase inversion.

In this paper, we report our results from large scale simulations of phase inversion in two-phase solid systems with dilatational misfit. Our study has been designed to overcome the specific shortcomings mentioned above. Thus, in our study, (a) elastic moduli of the phases may possess a cubic anisotropy, (b) elastic stress computations do not involve any approximations, and (c) system size is large. Further, in each set of simulations, we start with the same microstructures; this allows us to estimate quantitatively the dependence of phase inversion on modulus mismatch and elastic anisotropy.

${ }^{*}$ Corresponding author. Email: gururajan.mp@gmail.com

Philosophical Magazine

ISSN 1478-6435 print/ISSN 1478-6443 online (c) XXXX Taylor \& Francis

http://www.tandf.co.uk/journals

DOI: $10.1080 / 1478643 \mathrm{YYXxxxxxxx}$ 
This paper is organized as follows: In section 2, we present a brief summary of the phase tield model; the details of the formulation can be found elsewhere [8]. In Section 3, we illustrate phase inversion in both elastically isotropic and anisotropic systems, and describe a way of computing size at which phase inversion occurs. In Section 4, illustrate the effect of modulus mismatch and elastic anisotropy on phase inversion. We conclude the paper with a discussion and a summary.

\section{Modelling}

The diffuse interface formulation for the study of microstructural evolution in elastically stressed systems is well known (See for example [7,9-16]), and several of these studies are based on Fourier spectral techniques $[9,10,12-14]$. Ours is also a phase field model with a semi-implicit Fourier spectral implementation. We present a brief outline of our model; the details may be found in Ref. [8] (available online).

\subsection{Formulation}

Consider a microstructure in a binary $A-B$ system described by a composition field $c$ and consisting of two phases: A-rich $m$ and B-rich $p$ phases (which correspond to normalised compositions of unity and zero, respectively). Let the free energy of the system be given by

$$
F=N_{V} \int\left[A c^{2}(1-c)^{2}+\kappa(\nabla c)^{2}\right] d V+\frac{1}{2} \int \sigma: \varepsilon^{\mathrm{el}} d V
$$

where, $N_{V}$ is the number of atoms per unit volume, $\kappa$ is the gradient energy coefficient, $A$ is a constant (with dimensions of energy per atom) that sets the height of the energy barrier between the $p$ and $m$ phases, $\sigma$ is the elastic stress field, $\varepsilon^{e l}$ is the elastic strain, and the symbol ":" denotes tensor scalar product. We have assumed the gradient energy coefficient $\kappa$ to be a scalar constant, which leads to an isotropic interfacial energy.

The microstructural evolution in such a system is obtained by solving the following modified CahnHilliard equation:

$$
\frac{\partial c}{\partial t}=\nabla \cdot M \nabla \mu
$$

where, $M$ is the mobility, and $\mu$ is the chemical potential defined by given by the variational derivative of the free energy $F$ with respect to composition:

$$
\mu=\frac{\delta\left(F / N_{V}\right)}{\delta c}
$$

In this equation operator $\delta / \delta c$ represents the variational derivative with respect to composition.

Our phase field simulations are based on solving the diffusion equation 2 above, under the following assumptions:

(i) For any given composition distribution, the elastic equilibrium is obtained instantaneously.

(ii) Mobility $M$ is independent of the local state of stress.

(iii) Elastic stresses and strains obey Hooke's law:

$$
\sigma_{i j}=C_{i j k l} \varepsilon_{k l}^{\mathrm{e}}
$$

where, $\varepsilon^{\mathrm{el}}=\varepsilon-\varepsilon^{0}, \varepsilon_{i j}$ and $\varepsilon^{0}$ being the total strain and eigenstrain, respectively, and $C_{i j k l}$ is the elastic modulus tensor, which may have cubic anisotropy. 


$$
\begin{gathered}
C_{i j k l}(c)=C_{i j k l}^{\mathrm{e} f f}+\alpha(c) \Delta C_{i j k l} \\
\varepsilon_{i j}^{0}(c)=\beta(c) \varepsilon^{T} \delta_{i j}
\end{gathered}
$$

where, $\alpha(c)$ and $\beta(c)$ are scalar (interpolation) functions of composition, $C^{\text {eff } f}$ is the 'effective' moduli, $\Delta C=C_{i j k l}^{p}-C_{i j k l}^{m}$ is the difference between the elastic moduli of the $p$ and $m$ phases, $\delta_{i j}$ is the Kronecker delta, and $\varepsilon^{T}$ is the strength of the eigenstrain and indicates the change in lattice parameter with composition.

For each configuration (represented by the instantaneous composition field) with its associated eigenstrain field, we first solve the equation of mechanical equilibrium under zero prescribed (applied) stress. For this purpose, we use an iterative Fourier spectral technique obtained by modifying the stress-control via strain-control algorithm [17] (see also Ref. [18]) to systems with an eigenstrain field. Our formulation is similar to that of $\mathrm{Hu}$ and Chen [9] who extended the work of Khachaturyan et al [19]. However, Hu and Chen presented - and used - their algorithm to study systems under a prescribed homogeneous strain. Since we are interested in studying phase inversion under a zero applied stress, we have found it easier to modify the algorithm of Moulinec et al [17] for our study.

With this formalism, we simulate microstructural evolution as follows: For a given composition (and hence, eigenstrain) field $c(\mathbf{r}, t)$, a numerical solution to the equation of mechanical equilibrium yields elastic stress $(\sigma)$ and strain $(\varepsilon)$ fields. They, in turn, yield $\mu$ the chemical potential field through through Eq. 3 and Eq. 1. Substitution of $\mu$ in the Cahn-Hilliard equation (Eq. 2), and a numerical time-integration (over a time step $\Delta t$ ) yields $c(\mathbf{r}, t+\Delta t$ ), the composition field at the end of the time-step, on which this process is repeated. We use a semi-implicit Fourier spectral technique, due to Chen and Shen [20], for numerical integration of the Cahn-Hilliard equation. The (discrete) Fourier transforms needed for our calculations have been carried out using FFTW developed by Frigo and Johnson [21].

\section{$2.2 \quad$ Parameters}

We non-dimensionalise the diffusion equation in such a way that both $\kappa$ and $A$ take the value of unity, the equilibrium compositions of the $p$ and $m$ phases are at unity and zero respectively. Our calculations use a $1024 \times 1024$ grid in two dimensions with a grid spacing of $\Delta x=\Delta y=1$. For the time integration of the Cahn-Hilliard equation, we have used a time step of $\Delta t=0.5$ for the first 10000 time steps, and $\Delta t=1.0$ later.

For a system with cubic elastic constants, the circular averages of the Voigt constants $\left(\bar{C}_{11}, \bar{C}_{12}\right.$, and $\left.\bar{C}_{44}\right)$ can be related to the average shear modulus $G$, the Poisson's ratio $\nu$, and the anisotropy parameter $A_{Z}[22]$ using the following expressions:

$$
\begin{gathered}
G=\bar{C}_{44}, \\
\nu=\frac{1}{2} \frac{\bar{C}_{12}}{\bar{C}_{12}+\bar{C}_{44}},
\end{gathered}
$$

and,

$$
A_{Z}=\frac{2 \bar{C}_{44}}{\bar{C}_{11}-\bar{C}_{12}} .
$$

If $A_{Z}=1$, the elastic constants are isotropic; if $A_{Z}>1$ (or, $A_{Z}<1$ ), the $\langle 10\rangle$ and $\langle 11\rangle$ directions are 
elastic inhomogeneity ratio $\delta$ as the ratio of the shear modulus of the precipitate to that of the matrix: $\delta=\bar{C}_{44}^{p} / \bar{C}_{44}^{m}$.

For each system we have studied, we assume that $\nu^{p}=\nu^{m}$, and $A_{Z}^{p}=A_{Z}^{m}$; i.e., elastic anisotropy and Poisson's ratio are the same for both $p$ and $m$ phases. Thus, we need to specify $A_{Z}, \nu$ and $G$ for the $m$ phase, and the inhomogeneity parameter $\delta$ to completely characterise the elastic moduli.

In all our calculations, we have used $\nu^{p}=\nu^{m}=0.3, \varepsilon^{T}=0.01$, and $\alpha(c)=\beta(c)=c-c_{0}$. Moreover, the $p$ phase is always harder than the $m$ phase $(\delta>1)$. Displacement fields are iteratively refined until they converge to within $10^{-8}$.

The initial microstructure in all our simulations is shown in Figure 1: it consists of circular particles of the soft, A-rich $m$ phase (black) embedded in the hard, B-rich $p$ phase (white). The volume fraction of the $p$ phase is $\approx 60 \%$. It is possible to start with a microstructure with a uniform composition of $60 \%$ throughout the domain with a very small initial noise and study phase separation and inversion. However (and as we stated in the Introduction), in such a case, different combinations of $A_{Z}$ and $\delta$ would lead to different microstructures before phase inversion. This would make impossible to compare phase inversion across systems with different elastic parameters.

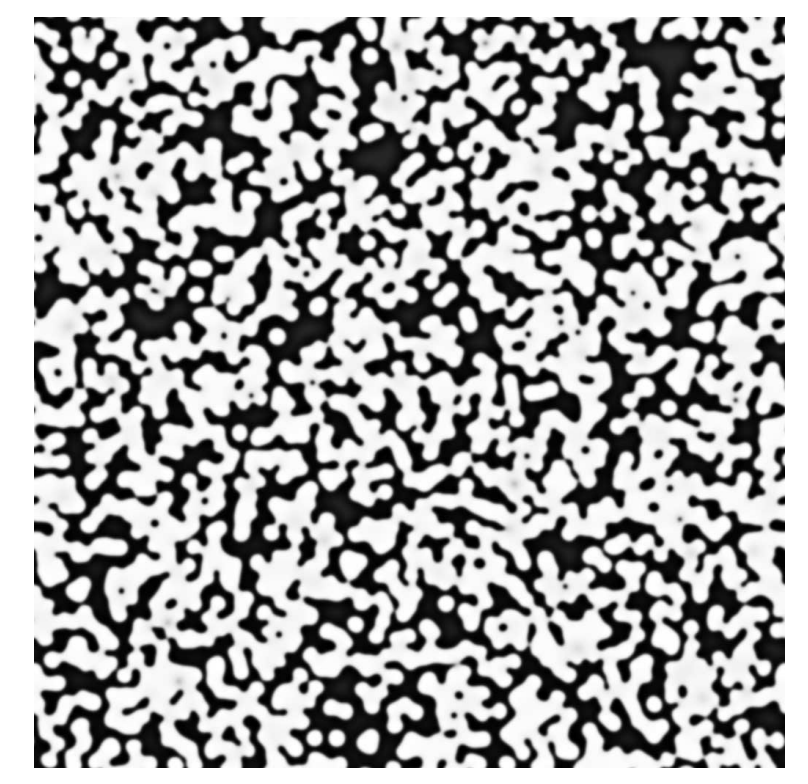

Figure 1. Initial microstructure used in all our simulations: $c_{0}=0.6$. The white phase is the hard phase.

\section{Results}

The initial microstructure in Fig. 1 evolves to yield those shown in Fig. 2 after (a) $t=500$ and (b) $t=8000$ for an elastically isotropic system with $\delta=2$. At $t=500$, the white phase (elastically hard $p$ phase) still constitutes the matrix in which islands of dark $m$ phase are embedded. By $t=8000$, however, the microstructure has undergone a phase inversion, with islands of the majority (white) phase embedded in the minority (dark) phase. In addition to visual inspection, we have used Hoshen-Kopelman clustercounting algorithm [23] to track phase inversion; operationally, for our two dimensional system, the phase which percolates both from left-to-right and from top-to-bottom in a microstructure is considered to be the matrix phase. Using such an approach (which would not work in three dimensions), we identify the time at which phase inversion takes place. At this time, we compute the characteristic length scale $\ell^{c}$, defined as the first zero of the circularly averaged pair correlation function. For each system, we report the average value of $\ell^{c}$ from three independent simulations. For example, the system shown in Figure 2 phase-inverted at $\ell^{c} \approx 30$. 


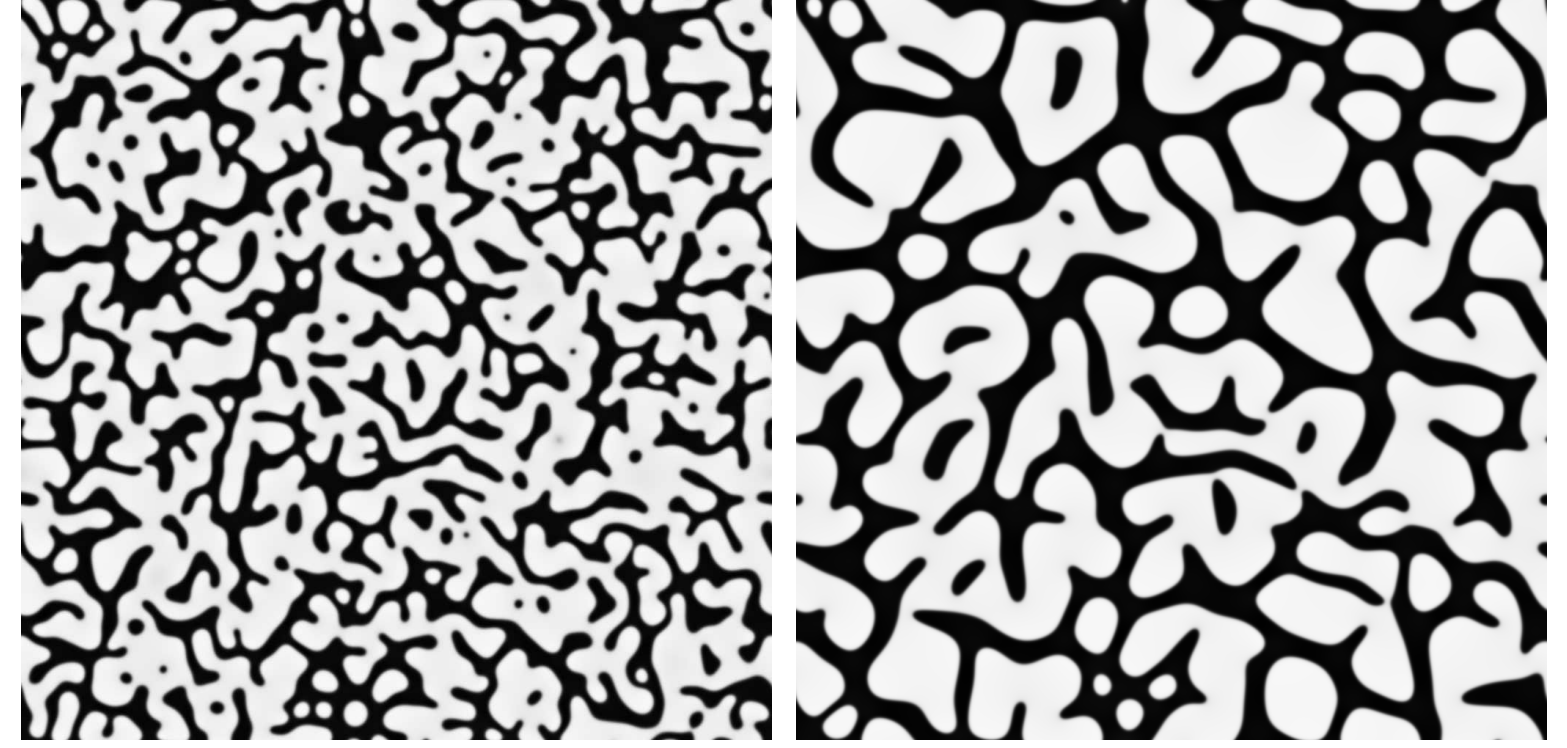

(a)

(b)

Figure 2. Phase inversion in an elastically isotropic system $\left(A_{Z}=1\right)$ with $\delta=2$ : microstructures after (a) 500 and (b) 8000 time units. While the white (hard) phase is percolating along both $x$ and $y$ directions in (a), the dark phase is percolating in (b): $c_{0}=0.6$.

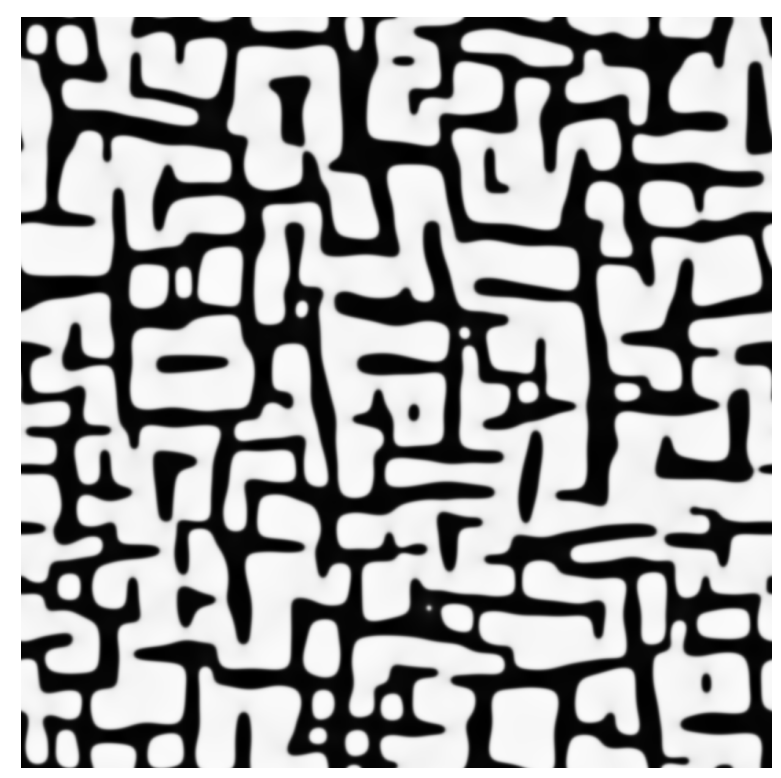

(a)

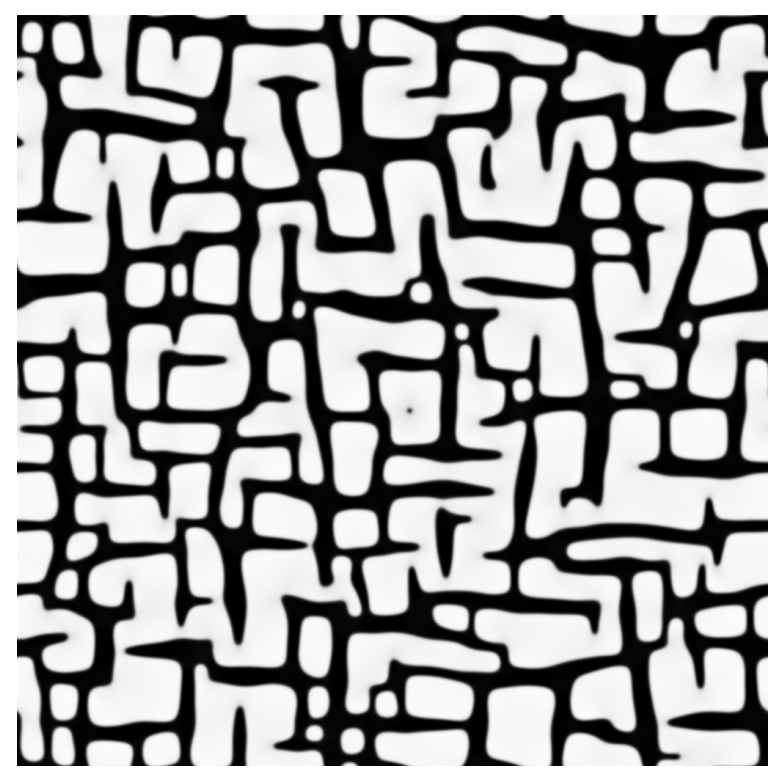

(b)

Figure 3. Effect of $\delta$ on phase inversion in an elastically anisotropic system $A_{Z}=3$. Microstructures after 4000 time units in systems with (a) $\delta=2$ and (b) $\delta=3: c_{0}=0.6$.

Figure 3 shows the microstructures at $t=4000$ in elastically anisotropic systems with $A_{Z}=3$ for two different levels of elastic inhomogeneity values: (a) $\delta=2$ and $\delta=3$. Both systems have undergone phase inversion to yield the $p$-phase (majority, hard phase, in white) as the embedded phase. We discern in these microstructures an alignment of the $p$ phase along the $\langle 10\rangle$ directions; the black channels of the minority $m$ phase run along these directions. In contrast, the microstructure for the elastically isotropic system in Figure 2 exhibits directionless, rambling black channels of the minority $m$ phase.

A closer examination of these microstructures also reveals that the $p$ phase is broken up more in the system with larger $\delta$. This implies that the system with the harder $p$ phase underwent phase inversion at a smaller size (or, earlier than $t=4000$ ). This may be rationalized by considering the driving force for 
phase inversion; with all else being the same, systems with harder $p$ phase would have a higher driving force, causing it to phase invert at smaller sizes.

Similarly, a comparison of Figure 2(b) and Figure 3(a) shows that, for the same inhomogeneity $(\delta=2)$, system with a cubic anisotropy has phase inverted at a smaller size. This is due to a geometric effect. Phase inversion may be thought of as a result of elongation and coalescence of the soft $(m)$ phase. In systems with cubic anisotropy, this elongation is along the soft $\langle 10\rangle$ directions, which facilitates coalescence. In isotropic systems, however, the elongation does not have any preferred direction, implying that an $m$ phase particle has to 'meander' before finding another meandering $m$ phase particle to coalesce with.

These broad conclusions are summarized more quantitatively in Figure 4, in which the length scale of phase inversion $\left(\ell^{c}\right)$ is plotted against elastic anisotropy $\left(A_{Z}\right)$ for different two different values of elastic inhomogeneity $(\delta=2$ and $\delta=3)$. The main conclusion is that both anisotropy $A_{Z}$ and inhomogeneity $\delta$ are important only at small values of $A_{Z}$. Increasing anisotropy beyond $A_{Z}=2$, leads only to small differences in the critical size for phase inversion.

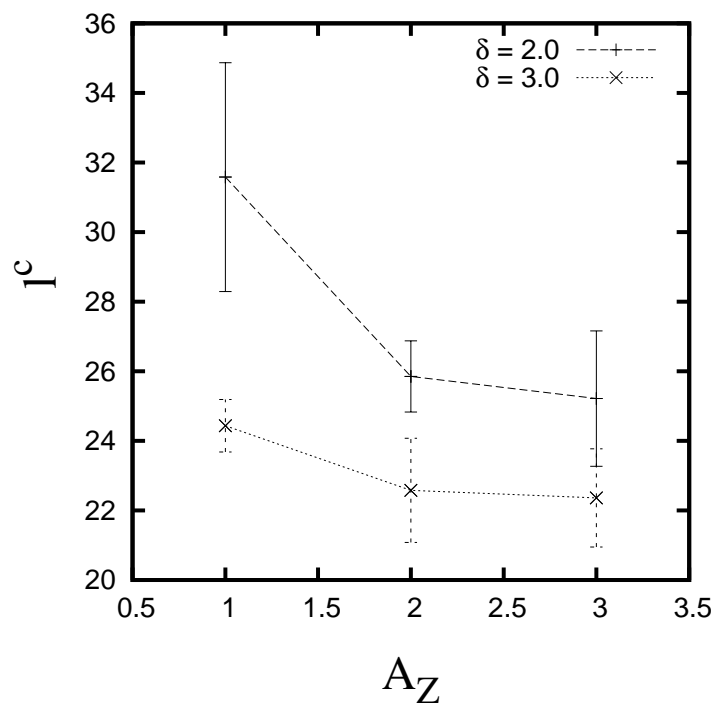

Figure 4. The effect of $A_{Z}$ on the characteristic length scale for phase inversion (for two different $\delta$ ). The data is averaged from three simulations on a system of composition $c_{0}=0.6$. The lines joining the data points are drawn only as a guide to the eye, while the error bar on the data points denotes the standard deviation.

\section{Discussion}

Phase inversion is reported to occur in several phase separating, viscoelastic polymer blends, and has been studied both experimentally and theoretically (see [24] and references therein). However, in the polymer literature, phase inversion is defined in several different ways. In some studies [25], with increasing volume fraction of one of the phases, if a previously continuous phase becomes discontinuous (thus switching the role of 'network' and 'particles' in a polymer blend), the volume fraction at which the switching occurs is known as 'phase inversion' point. In the work of Lazo and Scott [26], volume fraction of the phases is held a constant, and the initial microstructure with islands of the majority phase dispersed in a matrix of the minority phase, evolves into one in which the minority phase forms the dispersed phase.

Finally, some theoretical studies on polymer blends define phase inversion as that point at which "the role of the percolating majority phase and the non-percolating minority phase is interchanged between the low- and the high-viscosity portions of the liquid' [27]. This definition is similar to the one adopted in our study.

In polymer systems, phase inversion is a result of the flow of a less viscous, low volume fraction phase around a higher viscous, high volume fraction phase. In contrast, in this paper we have shown elastic stress driven phase inversion in solid systems, in which particles of a soft, minority $(m)$ phase elongate 
All the existing studies of elastic stress driven phase inversion employ approximate computations of elastic stresses; while Sagui et al [6] and Onuki and Nishimori [5] consider systems with small differences in $\Delta C$, Leo et al [7] do not incorporate the homogeneous strain in their calculations. Further, all the three studies are on isotropic systems. Finally, Sagui et al and Onuki and Nishimori spinodally decompose the microstructures, while Leo et al start with a very specific configuration. In view of these significant differences between these studies and ours, we do not attempt a critical comparison.

In each set of simulations (with different combinations of elastic moduli), we have used the same initial microstructure, with elastically soft $m$ phase particles embedded in the $p$ phase which is also the majority phase. As noted earlier, using the same initial microstructure allows a quantitative comparison of different systems. Further, we note that an initial microstructure in which the majority phase is the matrix phase is not unexpected; for example, in the absence of elastic stresses, spinodal decomposition in off-symmetric alloys is known to give rise to microstructures with a dispersed minority phase [28].

Since phase inversion is driven by elastic stresses, an increase in the the magnitude of elastic stresses can be expected to lead to phase inversion at smaller sizes (i.e., at smaller value of $\ell^{c}$ ). Our simulation results (not shown here) show that this is indeed the case. In addition, phase inversion is also influenced by the inhomogeneity $\delta$ and elastic anisotropy $A_{Z}$ (as shown in Figure 4). We have rationalized this observation (in Section 3) in terms of the driving force for phase inversion and the geometry of elongation and coalescence of the soft $m$ phase.

All the results we have presented in this study are for systems in which $f_{m}$, the soft (minority) phase volume fraction, is 0.4 . With lower $f_{m}$, the soft phase particles have to elongate more before they can coalesce to form a percolating network. This implies that phase inversion would occur at larger sizes in systems with lower $f_{m}$. This is indeed the case; in our simulations with $f_{m}=0.3$, it took too long before phases underwent an inversion. By this time, however, the number of hard phase particles was too small to allow us to extract a statistically meaningful and reliable value of $\ell^{c}$; therefore, we have not reported any data for this system.

\section{Summary}

Elastic stress-driven phase inversion is observed in multiparticle simulations in elastically inhomogeneous, coherent binary alloys. The characteristic length scale at which phase inversion occurs is decreases with an increase in the elastic inhomogeneity in elastic anisotropy; this influence is stronger at lower values of elastic anisotropy. $\ell^{c}$ also increases with a decrease in the volume fraction of the soft, minority phase. These trends have been rationalised in terms of the combined effect of the driving force for phase inversion and geometry.

\section{Acknowledgements}

We thank Saswata Bhattacharyya for useful discussions, and the Volkeswagen Foundation for financial assistance.

\section{References}

[1] D. M. Barnett, J. K. Lee, H. I. Aaronson, and K. C. Russell. The strain energy of a coherent ellipsoidal precipitate. Scripta Metallurgica, 8:1447-1450, 1974.

[2] R Sankarasubramanian, C S Jog, and T A Abinandanan. Symmetry-breaking transitions in equilibrium shapes of coherent precipitates: effect of elastic anisotropy and inhomogeneity. Metallurgical and Materials Transactions A, 33A:1083-1090, April 2002.

[3] Hajime Tanaka and Takeaki Araki. Phase inversion during viscoelastic phase separation: roles of bulk and shear relaxation moduli. Physical Review Letters, 78(26):4966-4969, June 1997.

[4] Hajime Tanaka. Viscoelastic model of phase separation. Physical Review E, 56(4):4451-4462, October 1997.

[5] Akira Onuki and Hiraku Nishimori. Anomalously slow domain growth due to a modulus inhomogeneity in phase-separating alloys. Physical Review B, 43(16):13649-13652, June 1991.

[6] Celeste Sagui, A M Somoza, and Rashmi C Desai. Spinodal decomposition in an order-disorder phase transition with elastic fields. Physical Review E, 50(6):4865-4879, December 1994. 
[7] P. H. Leo, J. S. Lowengrub, and H. J. Jou. A diffuse interface model for microstructural evolution in elastically stressed solids. Acta Materialia, 46(6):2113-2130, 1998.

[8] M P Gururajan. Elastic inhomogeneity effects on microstructures: a phase field study. PhD thesis, Department of Metallurgy, Indian Institute of Science, Bangalore 560012 INDIA, January 2006. Available at http://imechanica.org/node/440.

[9] S. Y. Hu and L. Q. Chen. A phase-field model for evolving microstructures with strong elastic inhomogeneity. Acta Materialia, 49:1879-1890, 2001.

[10] Jingzhi Zhu, Long-Qing Chen, and Jie Shen. Morphological evolution during phase separation and coarsening with strong inhomogeneous elasticity. Modelling and Simulation in Materials Science and Engineering, 9:499-511, 2001.

[11] Yu U Wang, Yongmei M Jin, and Armen G Khachaturyan. Phase field microelasticity theory and simulation of multiple voids and cracks in single crystals and polycrystals under applied stress. Journal of Applied Physics, 91(10):6435-6451, May 2002.

[12] Yu U Wang, Yongmei M Jin, and Armen G Khachaturyan. Three-dimensional phase field microelasticity theory of a complex elastically inhomogeneous solid. Applied Physics Letters, 80(24):4513-4515, June 2002.

[13] Y M Jin, Y U Wang, and A G Khachaturyan. Three-dimensional phase field microelasticity theory and modeling of multiple cracks and voids. Applied Physics Letters, 79(19):3071-3073, November 2001.

[14] Yu U Wang, Yongmei M Jin, and Armen G Khachaturyan. Phase field microelasticity theory and modeling of elastically and structurally inhomogeneous solid. Journal of Applied Physics, 92(3):1351-1360, August 2002.

[15] H J Jou, P H Leo, and J S Lowengrub. Microstructural evolution in inhomogeneous elastic media. Journal of Computational Physics, 131:109-148, 1997.

[16] D. Y. Li and L. Q. Chen. Shape evolution and splitting of coherent particles under applied stresses. Acta Materialia, 47(1):247-257, 1999.

[17] J. C. Michel, H. Moulinec, and P. Suquet. Effective properties of composite materials with periodic microstructure: a computational approach. Computer Methods in Applied Mechanics and Engineering, 172:109-143, 1999.

[18] A Anthoine. Derivation of the in-plane elastic characteristics of masonry through homogenization theory. International Journal of Solids and Structures, 32(2):137-163, 1995.

[19] A. G. Khachaturyan, S. Semenovskaya, and T. Tsakalakos. Elastic strain energy of inhomogeneous solids. Physical Review B, 52(22):15909-15919, December 1995

[20] L Q Chen and Jie Shen. Applications of semi-implicit Fourier-spectral method to phase field equations. Computer Physics Communications, 108:147-158, 1998.

[21] Matteo Frigo and Steven G. Johnson. The design and implementation of FFTW3. Proceedings of the IEEE, 93(2):216-231, 2005. special issue on "Program Generation, Optimization, and Platform Adaptation"; also see http://www.fftw.org/.

[22] I. Schmidt and D. Gross. Directional coarsening in Ni-base superalloys: analytical results for an elasticity based model. Proceedings of Royal Society (London) A, 455:3085-3106, 1999.

[23] J Hoshen and R Kopelman. Percolation and cluster distribution. I. Cluster multiple labeling technique and critical concentration algorithm. Physical Review B, 14(8):3438-3445, October 1976.

[24] Takashi Taniguchi and Akira Onuki. Network domain structure in viscoelastic phase separation. Physical Review Letters, 77(24):49104913, December 1996.

[25] C Z Chuai, K Almdal, and J Lyngaae-Jørgensen. Phase continuity and inversion in polystyrene/poly (methyl methacrylate) blends. Polymer, 44:481-493, 2003.

[26] N D B Lazo and C E Scott. Morphology development during phase inversion in isothermal, model experiments: steady simple-shear and quiescent flow fields. Polymer, 42:4219-4231, 2001.

[27] D Sappelt and J Jaeckle. Percolation inversion in spinodal decomposition of mixtures with strong kinetic asymmetry. Polymer, 39(21):5253-5256, 1998

[28] Y Wang, L Q Chen, and A G Khachaturyan. Kinetics of strain-induced morphological transformation in cubic alloys with a miscibility gap. Acta Metallurgica et Materialia, 41(1):279-296, 1993. 